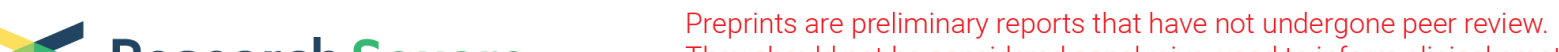 $\begin{array}{ll}\text { Research Square } & \text { They should not be considered conclusive, used to inform clinical practice, } \\ \text { or referenced by the media as validated information. }\end{array}$
}

\section{Case report: A Re-operation Case of Asymptomatic Left Giant Pheochromocytoma and Literature Review}

\section{Wei Dai}

The Affiliated Hospital of Guangdong Medical University

\section{Xiaoguang Liu}

The Affiliated Hospital of Guangdong Medical University

Jialiang Dai

The Affiliated Hospital of Guangdong Medical University

\section{Minqiang Chen}

The Affiliated Hospital of Guangdong Medical University

\section{Jian Mo}

The Affiliated Hospital of Guangdong Medical University

\section{Ming Chen}

The Affiliated Hospital of Gaungdong Medical University

\section{Xiaoyu Tan}

The Affiliated Hospital of Guangdong Medical University

\section{Xiaofeng Chen}

The Affiliated Hospital of Guangdong Medical University

\section{Qiongyan Lin}

The Affiliated Hospital of Guangdong Medical University

\section{Jie Wang}

Sun Yat-Sen Momorial Hospital,Sun Yat-Sen University https://orcid.org/0000-0002-6456-1101

\section{Shuo Fang}

The Seventh Affiliated Hospital Sun Yat-sen University

Nianping Chen ( $\sim$ npc0413@hotmail.com )

The Affiliated Hospital of Guangdong Medical University

\section{Case report}

Keywords: Pheochromocytoma, giant, asymptomatic, re-operation, catecholamine

Posted Date: February 28th, 2020

DOI: https://doi.org/10.21203/rs.2.24809/v1 
License: (c) (i) This work is licensed under a Creative Commons Attribution 4.0 International License. Read Full License 


\section{Abstract}

Introduction: Pheochromocytoma (PCC) is a rare tumor which derives from adrenal medulla, when maximum diameter of pheochromocytoma is greater than $10 \mathrm{CM}$, it is divided into Giant pheochromocytoma(GPCC), which is extremely rare and usually asymptomatic.Clinically, a huge and asymptomatic adrenal pheochromocytoma was usually misdiagnosed as other types of tumors, which result in notable increase of complication rates and death rates.

Case presentation:In this case report, we described a clinically asymptomatic GPCC patient. Diagnosis:According to computed tomography (CT) scan, nuclear magnetic resonance(MR) scan, the patient is initially diagnosed as liposarcoma.After laparotomy, biochemical detection of catecholamine (CA) intermediate metabolites methoxyepinephrine (MN) and methoxy norepinephrine (NMN) and the pathological examination, diagnosis of GPCC was obtained. Intervention囚The laparotomy was suspended and a diagnosis of pheochromocytoma was confirmed, because of unstable blood pressure and hypertension during separating and moving the mass during surgery. After consultation of multidisciplinary team, adequate preoperative preparation was conducted according to the procedure of PCC surgical preparation. By the end of the regular phenoxybenzamine and intravenous fluids treatment for three weeks, the blood pressure of the patient was kept at an acceptable average. Therefore, the patient underwent the operationagain for radical resection.

Outcome: After the operation, CA was basically normal in rechecking process, and the tumor was successfully removed. Pathological diagnosis of the mass after operation: Immunohistochemical resulted conformed to (epigastrium) pheochromocytoma. Immunohistochemistry: $\mathrm{CgA}(+)$, Inhibin-a(-), ki$67(<1 \%+)$, Syn $(+)$. Pheochromocytoma had the definite pathological diagnosis.

Conclusion: GPCC has the high diagnostic identification difficulty, it should be combined with imaging examination and biochemical measurement to identify. Before operation, the detailed imageological examination provides important reference for excision and surgical planning. Individualized and multidisciplinary cooperation of management strategy in perioperative period should be recommended. Patients should conduct long-term follow-up.

\section{Introduction}

Pheochromocytoma (PCC) is a rare tumor which derives from adrenal medulla, leading to aseries of clinical syndromes through synthesis or release of catecholamine (CA), with main manifestations of hypertension and its complications. ${ }^{[1]}$ Clinically, most adrenal tumors larger than $7 \mathrm{~cm}$ are often found to be dysfunctional malignancies.. PCC could secrete catecholamines continuously or intermittently with different proportion. Adrenergicreceptor is widely distributed in a variety of body tissues and cells, leading to clinical manifestations and individual variations. A small proportion of patients can be asymptomatic. Clinically, a huge and asymptomatic adrenal pheochromocytoma was usually misdiagnosed as other types of tumors, which result in notableincrease of complication rates and death rates. Although surgery 
remains the primary therapy option for patients with large adrenal tumor, it is still a huge challenge for abdominal surgeons because of difficult exposure, high morbidity rates of cardiovascular complication, metastasis and invasion of huge tumors. In this case report, we described a clinically asymptomatic GPCC patient who had surgery to remove a $18 \mathrm{~cm} \times 21 \mathrm{~cm} \otimes 19 \mathrm{~cm}$ huge tumor mass in November 2017, with a good postoperative recovery and no complications. Now it is reported as follows.

\section{Case Presentation}

\subsection{Disease history and auxiliary examination囚}

The patient was a 30-year-old man with an abdominal mass more than 4 months $囚$ examined by abdominal B-ultrasound in outpatient pharmacy of our hospital and suggested an occupying lesion in abdomen. Then retroperitoneal tumors was diagnosed and admitted in the hospital. The patient had no abdominal distension, abdominal pain, constipation, diarrhea, dizziness, headache, palpitation, shortness of breath, back pain, or progressive emaciation, his previous medical history is without any paroxysm hypertension, diabetes, surgery or trauma. The patient's mother and father are healthy. No family history of diseases or tumors. Physical examinations: Temperature $37.2^{\circ} \mathrm{C}$, Heart rate 63 times $/ \mathrm{min}$, Respiration 18 times/min $\otimes$ Blood pressure $120 / 80 \mathrm{mmHg}$. A crucial finding on physical examination is obvious protrusion mass in upper left abdomen $\$ approximately $15 \mathrm{~cm} \times 12 \mathrm{~cm}$ in size. The abdomen is supple without epigastric varicose veins or tenderness, rebound tenderness. Some additional tests were performed after admission, such as "three great regular tests", second liver two half-and-half detect, three indexes before blood transfusion, four coagulation tests, 36 biochemical indexes, tumor markers , Chest radiographs, and showed no abnormalities. Electrocardiograms: obvious sinus bradycardia complicated witharrhythmia, junctional escapebeat, 44 times/min. Ambulatory electrocardiogram: 1 . sinus rhythm, longest R-R<2.0s; 2 . occasional atrial premature; 3. ST-T change. Important image detections, such as upperand lower abdominal CT囚plain and enhanced囚+CTA+CTV (Fig.1) showed: 1. left upper abdominal and retroperitoneal huge mass was considered as malignant lesion, doubtful of myxoid liposarcoma. 2. considered as intrahepatic scattered small cyst. 3. CTA showed compression and displacement of splenic artery and left renal artery, blood vessels of superior mesenteric artery and small branches of splenic artery appeared on the substantial part of tumor edge. 4. CTV showed compression and displacement of splenic vein, but without filling defect. Superior abdominal MR suggested a irregular mass in left upper-middle abdomen, which is about $18 \mathrm{~cm} \times 18 \mathrm{~cm} \times 15 \mathrm{~cm}$ in size, with Heterogeneous signal (Fig.2). Multiplenecrosis and cystic lesions were detected in the mass. The stomach was compressed leading to displacement to the front and the right, the spleen was compressed to the left lateral, and the spleen, pancreas and the left kidney were compressed downward. Lesions showed significant but heterogenous enhancement and could not be separated from the pancreas and spleen $₫$ but showed clear boundaries with other surrounding structures. The diagnosis of upper left retroperitoneal malignant mesenchymoma was made by the radiography, possibly liposarcoma, leading to displacement of the spleen and the left kidney. Based on this information and the patient's medical history, radical resection is the first choice. 


\subsection{First operation.}

Temporary pacemaker was used to treat decreased heart rate of the patient after hospitalized. Subsequently, the patient was subjected to laparotomy and a diagnosis of pheochromocytoma was evacuated on October 10, 2017. Unstable blood pressure and hypertension (up to $330 / 160 \mathrm{mmHg}$ ) during separating and moving the mass leaded to suspension of surgery and is admitted to the intensive care unit.

Postoperative auxiliary examination: adrenocorticotrophic hormone $10.56 \mathrm{pg} / \mathrm{mL}$. Determination of

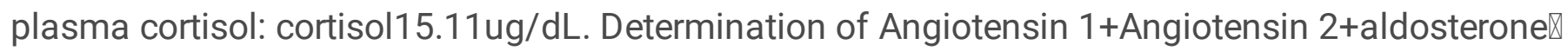
aldosterone77.17pg/mI plasma renin activity $6.76 \mathrm{ng} / \mathrm{mL} / \mathrm{hr}$ \angioten $\sin 18.16 \mathrm{ng} / \mathrm{mL}$ \angiotensin $11.40 \mathrm{ng} / \mathrm{mL}$ 冈angiotensin II 58.92pg/mL (October 19, 2017). Determination of amylase (Rate method) : amylase $320 \mathrm{U} \mathbb{\mathrm { L }}$ (October 20, 2017). Determination of urinary potassium (24 hours): potassium $56.30 \mathrm{mmol} / 24 \mathrm{~h}$ (October 21, 2017).

After consultation of multi-disciplinary team, adequate preoperative preparation was conducted according to the procedure of PCC surgical preparation. By the end of the regular phenoxybenzamine and intravenous fluids treatment for three weeks, the blood pressure of the patient was kept at an acceptable average $(100-120 \mathrm{mmHg} / 60-80 \mathrm{mmHg})$, heart rate60-90 beats / min . Erratic blood pressure only appeared before sleep (Systolic pressure100-150 mmHg). Therefore, the patient underwent the operation again on November 15, 2017.

\subsection{The second surge}

Abdominal exploration showed slight adhesion, and large cystic solid mass in the left abdomen, about the size of $18 \mathrm{~cm} \nabla 21 \mathrm{~cm} \nabla 19 \mathrm{~cm}$, moderate hardness, close adhesion with greater curvature and jejunum, with invasion of pancreatic tail, splenic artery, splenic vein. No obvious metastasis was found in liver, kidney, hepatic hilar region or mesenteric roots. Slight fluctuation of blood pressure occurred during abdominal exploration. Intraoperative diagnosis is left adrenal pheochromocytoma and resection of left giant adrenal pheochromocytoma + splenectomy + partial resection of the pancreas tail+ enterolysis were performed in surgery: released the adhesion intestinal canal, incised the gastrocolic ligament, fully exposed the pancreas $\$ and separated the mass over the pancreas. Then the splenic artery and splenic vein were ligatured, a part of pancreatic tails was removed. A drag hook was used to pull the abdominal wall towards the left side, while the spleen was dragged towards right side, showing external lienorenal ligament. An ultrasound knife was used for cutoff. It was separated towards the fundus of stomach from 
the greater curvature to cut off gastrosplenic ligament. Also, short gastric vessel was ligated one by one, dissociating the upper half and inside margin of left kidney, revealing the adrenal gland, carefully separating from the adrenal gland and ambient adipose tissues, and completely dissociating and cutting off the entire tumor. In the dissociated process, blood pressure was fluctuated at $60 / 40 \mathrm{mmHg}$ $230 / 130 \mathrm{mmHg}$. Heart rate was fluctuated at times/second. Body of pancreas and spleen were combined with the left adrenal gland to cut off and move out of the abdominal cavity. After the posterior peritoneum wound surface fully stopped bleeding, a drainage tube was placed at the spleen fossa. Instruments and gauze were counted without errors. Abdomen was closed layer by layer. The operation process was successful and anesthesia was satisfactory. Amount of bleeding in operation was about $500 \mathrm{~mL}$. After the operation, the patient returned to the ward. A specimen was cut off for conventional pathological examination.(Figure 3)

\subsection{Postoperative conditions and pathological results.}

After the operation, the patient was sent to ICU for nursing. In the second day after operation, the patient had a fever. After the patient accepted anti-infective therapy, vital signs gradually tended to be smooth. After the third week of operation, CA was basically normal in rechecking process. Considering that the tumor was cut off successfully, the patient was allowed to discharge from the hospital. Pathological diagnosis of the mass after operation: 1. Immunohistochemical resulted conformed to (epigastrium) pheochromocytoma, showing invasion of suspicious diolame; 2. Spleen and pancreatic tissue showed chronic inflammation and atrophy changes, showing no tumor invasion; there was no tumor in surrounding adipose tissues. Immunohistochemistry: $\mathrm{CgA}(+)$, Inhibin-a(-), ki-67(<1\%+), Syn(+). Pheochromocytoma had the definite pathological diagnosis. (Figure 4). It hasn't relapsed after 8-month follow-up.

\section{Discussion}

Pheochromocytoma is a rare tumor that is originated from adrenal medulla. It is estimated that the morbidity of global people is $0.005-0.1 \% .^{[2]}$ The clinical symptoms of the typical pheochromocytoma are reflected in paroxysmal headache, perspiration and palpitation. Continuous hypertension is generally considered as one of important clinical performances, while in all pheochromocytoma cases, only a few patients will have normal blood pressure or have no symptoms. The morbidity of the asymptomatic pheochromocytoma is estimated as $21 \%$ of PCC total morbidity. ${ }^{[1]}$ When maximum diameter of pheochromocytoma is greater than 10CM, it can be divided into Giant pheochromocytoma(GPCC), which is extremely rare and asymptomatic. ${ }^{[3]}$ By existing English literatures, We speculate the reason why such pheochromocytoma patients have no symptoms is that the giant tumor center has the bigger necrosis area for insufficient nutrition and blood supply, thus the cell number of catecholamine is reduced. At the 
same time, more tumor stroma and desmoplasia will form the saccular structure. Catecholamine and metabolite generated by tumors will be stored in it. As conducting the resection operation, due to touch extrusion and tumor separation, such a saccular structure is destroyed. Lots of catecholamine will be released in blood, resulting in hypertensive crisis. ${ }^{[4-6]}$

In this case, the patient had no obvious continuous hypertension, but only had the vague abdominal sign. The abdominal CT only reminded the huge tumor in the left upper abdominal cavity and behind peritoneum. The neurogenic tumor (including pheochromocytoma only accounted for $10 \%-20 \%$ of primary retroperitoneal neoplasma. It was difficult to distinguish and identify in imageological examination. ${ }^{[7]}$ As a result, even if we had a doubt in early stage, we never diagnosed it as pheochromocytoma. Before the first operation, the patient had no clinical symptoms. By combining with hypertensive crisis of $330 / 160 \mathrm{mmHg}$ in the operation and biochemical measurement, it conformed to clinical diagnosis of GPCC. According to preoperative preparation scheme of PCC, operation in the second time was conducted. Pathology of the submittal specimen was finally confirmed as GPCC.

For GPCC, biochemical measurement and image data are two important methods of definite diagnosis. Biochemical measurement is used for qualitative diagnosis. $\mathrm{MN}$ and $\mathrm{NMN}$ are intermediate metabolites of epinephrine(E) and noradrenaline(NE) respectively,they have the longer half-life period in plasma and the small plasma concentration fluctuation. Therefore, the specificity and the sensibility are better than catecholamine measurementas diagnosing pheochromocytoma, but the positive rate is only $20 \%$,blood or urine $\mathrm{NE}, \mathrm{E}$ and dopamine(DA) measurement can be used for auxiliary diagnosis. In addition, the terminal metabolite vanillyl mandelic acid (VM-A) can be used for measurement to diagnosis, but the specificity is $86 \%-99 \%$, blood and urine CA levels should be measured simultaneously for definitive diagnosis. ${ }^{[8]}$ Imaging examination is used for localization diagnosis (Table 1). The sensibility of abdominal CT scan or magnetic resonance imaging (MRI) for pheochromocytoma is $90-100 \%$. Specificity is $70-80 \% .{ }^{\left[{ }^{2}\right]}$ Besides, ${ }^{131}$ I-MIBG is a very sensitive tool to detect pheochromocytoma, but as using this method by alone, its sensitivity is only $83.7 \%$. If it is combined with the plasma noradrenaline, sensitivity is up to $97.1 \%$. Noradrenaline from urine/MIBG is $96.6 \%$. Noradrenaline in urine/MIBG is $95.3 \%$. Blood platelet noradrenaline/MIGB is even up to $100 \% .{ }^{[9]}$ As a result, if there is suspected diagnosis of pheochromocytoma in clinics and catecholamine is kept in the normal range, we advocate conducting MIBG scanning.

For GPCC, once it makes a definite diagnosis and positioning, the first treatment should be operative excision. Early, suitable and appropriate acceptance of adrenergic receptor before operation can avoid from serious complications. Even if there is no official guide about blocking drug types, the currently used 
drugs mainly include a adrenergic retardant and calcium antagonist. The main objectives aim to normalize hemodynamics, including blood pressure, heart rate and liquid condition in blood vessels. Preoperative average treatment time is 2-6 weeks. a-receptor retardant including phenoxybenzamine and doxazosin is recommended as the front-line drugs. Calcium antagonist including nifedipine and amlodipine acts as the replaced drug or drug combination. Furthermore, $\beta$-receptor retardant is used to control tachycardia. In this case, phenoxybenzamine was used. ${ }^{[10]}$

Considering specificity of GPCC, we conducted MDT consultation before the second operation. According to the patient's situation, a-receptor retardant use scheme, fluid infusion scheme and operation mode were formulated individually, laying a foundation on success of operation. For the selection of the operation mode, multiple guides recommend to use excision of laparoscopic surgery. Because the GPCC tumor exceeds $9 \mathrm{~cm}$, the open operation is relatively safe to patients. The detailed imageological examination before operation can provide the important reference for resectable evaluation and surgical planning, such as abdominal enhanced CT+CTA+ CTV, abdominal enhanced MR and 3D visualized tumor reconstruction technology. In the surgical procedure, cooperation between the anesthesia team and surgeon will be good for reducing hypertensive crisis and risks caused by blood vessel complications. In the operation process, the patient's blood pressure, heart rate, central venous pressure and pulmonary wedge pressure should be continuously supervised. In early stage, surgeons block tumor vein to reduce secretion of catecholamine, while anesthesia team uses the suitable vasoactive agent to stabilize haemodynamics. Such coordination can reduce hypertensive crisis in operation to capacity. ${ }^{[11]}$

Lots of pheochromocytoma is a benign tumor, while the patients with malignant tumor account for $10 \%$. With the increase of tumor size, the malignant probability is also increasing. Five-year survival rate of benign tumors is $95 \%$, while that of malignant tumors is $5 \%$. At present, GPCC has no specific detection to accurately remind malignant lesions, thus postoperative follow-up is also important. The patient should recheck for once-twice every year. ${ }^{[12]}$ If it is malignant, ${ }^{131}$ I-MIBG treatment should be considered. ${ }^{[13]}$

\section{Conclusion}

4.1. GPCC has the high diagnostic identification difficulty. When imageological examination shows a retroperitoneal giant tumor in the epigastrium of left side or right side, there is the possibility of GPCC区 should be combined with biochemical measurement to identify. If possible, ${ }^{131}$ I-MIBG detection should be conducted for further definite diagnosis.

4.2. Before operation, the detailed imageological examination provides important reference for excision and surgical planning, recommending abdominal enhanced CT+CTA+ CTV, abdominal enhanced MR and 
3D visualized tumor reconstruction technology.

4.3. Individualized and multi-disciplinary cooperation of management strategy in perioperative period should be recommended.

4.4. In view of difficulty in identifying benign and malignant GPCC, it suggests that patients should conduct long-term follow-up.

\section{Abbreviations}

PCC=Pheochromocytoma, GPCC=Giant pheochromocytoma, $\mathrm{CT}=$ computed tomography, MRI=magnetic resonancelmaging, $\mathrm{CA}=$ catecholamine, $\mathrm{MN}=$ methoxyepinephrine, $\mathrm{NMN}=$ methoxy norepinephrine, $\mathrm{CTA}=$ computed tomography angiography, $\mathrm{E}=$ epinephrine, $\mathrm{NE}=$ noradrenaline, $\mathrm{DA}=$ dopamine, $\mathrm{VM}$ A=vanillyl mandelic acid.

\section{References}

[1]. Adler JT, Meyer-Rochow GY, Chen H, et al. Pheochromocytoma: current approaches and future directions. Oncologist. 2008 Jul;13(7):779-93.

[2]. Wang HL, Sun BZ, XU JZ, Lei WF, Wang XS. Undiagnosed giant cystic pheochromocytoma: A case report Oncol Lett. 2015 Sep; 10(3): 1444-1446.

[3]. Maharaj R, Parbhu S, Ramcharan W,et al. Giant cystic pheochromocytoma with low risk of malignancy: a case report and literature review. Case Rep Oncol Med.2017;2017ه4638608.

[4]. Li CF, Chen YS, Wang WT, Teng LC. A case of clinically silent giant right pheochromocytoma and review of literature. Can Urol Assoc J. 2012 Dec; 6(6): E267-E269.

[5]. Munakomi S, Rajbanshi S, Adhikary PS. Case Report: A giant but silent adrenal pheochromocytoma a rare entity Version 1. F1000Res. 2016; 5: 290. 
[6]. Muchuweti D, Muguti ED, Mbuwayesango BA, Mungazi SG,Makunike-Mutasa R. Diagnostic and surgical challenges of a giant pheochromocytoma in a resource limited setting-A case report. Int J Surg Case Rep. 2018; 50:111-115.

[7]. Scali EP, Chandler TM, Heffernan EJ, Coyle J囚Harris AC\van Chang SD. Primary retroperitoneal masses: what is the differential diagnosis? Abdom Imaging. 2015 Aug;40(6):1887-903.

[8]. Lenders JW, Pacak K, Walther MM, et al.Biochemical diagnosis of pheochromocytoma: which test is best? JAMA. 2002 Mar 20;287(11):1427-34.

[9]. Guller U, Turek J, Eubanks E, Delong ER, Oertli D, van Feldman JM.Detecting Pheochromocytoma: Defining the Most Sensitive Test. Ann Surg. 2006 Jan; 243(1): 102-107.

[10]. Isaacs M, Lee P.Preoperative alpha-blockade in phaeochromocytoma and paraganglioma:is it always necessary?Clin Endocrinol (Oxf). 2017 Mar;86(3):309-314.

[11]. Därr R, Lenders JWM, Hofbauer LC, Naumann B, Bornstein SR,van Eisenhofer G.

Pheochromocytoma - update on disease management. Ther Adv Endocrinol Metab. 2012 Feb; 3(1): 1126.

[12]. Ambati D, Jana K, Domes T. Largest pheochromocytoma reported in Canada: A case study and literature review. Can Urol Assoc J. 2014 May-Jun; 8(5-6): E374-E377.

[13]. Reisch N, Peczkowska M, Januszewicz A, Neumann HP. Pheochromocytoma: presentation, diagnosis and treatment. J Hypertens. 2006;24:2331-9.

\section{Declarations}

Availability of data and materials:

All data generated or analyzed are included in this published article. 
Contributions:

All authors participated in the patient's care. WD, XL, JM and QL performed the surgeries described in this report. MingC, XT and XC designed for surgery and preparation. JW, SF and NC constructed the conception and design of this report. JD and MingqiangC prepared the draft of the manuscript. All authors read and approved the final manuscript.

Acknowledgements:

Not applicable.

Funding:

None.

Ethics approval and consent to participate:

Not applicable.

Consent for publication:

The patient provided written consent to publish this case report.

\section{Competing interests:}

The authors declare that they have no competing interests.

\section{Table 1}

Table 1. Biochemical tests 


\begin{tabular}{|c|c|c|}
\hline Measured value & Reference range & Units \\
\hline Test item (24hr Urine Biochemical & & \\
\hline Adrenalineand, $24 \mathrm{hr}$ & $2-24$ & $\mathrm{mcg} / 24 \mathrm{~h}$ \\
\hline Norepinephrine, $24 \mathrm{hr}$ & $15-100$ & $\mathrm{mcg} / 24 \mathrm{~h}$ \\
\hline Dopamine, $24 \mathrm{hr}$ & $52-480$ & $\mathrm{mcg} / 24 \mathrm{~h}$ \\
\hline Urine creatinine & $0.63-2.50$ & $\mathrm{~g} / 24 \mathrm{~h}$ \\
\hline Vanilmandelic acid & 24.98- 70.2 & $\mu \mathrm{mol} / 24 \mathrm{~h}$ \\
\hline Total & $26-121$ & $\mathrm{mcg} / 24 \mathrm{~h}$ \\
\hline Test item (separated plasma) & & \\
\hline Total methoxyepinephrine & $<=205$ & $\mathrm{pg} / \mathrm{mL}$ \\
\hline methoxynorepinephrine & $<=148$ & $\mathrm{pg} / \mathrm{mL}$ \\
\hline Metanephrine & $<=57$ & $\mathrm{pg} / \mathrm{mL}$ \\
\hline Dopamine & $<30$ & $\mathrm{pg} / \mathrm{mL}$ \\
\hline Noradrenaline & 217-1109 & $\mathrm{pg} / \mathrm{mL}$ \\
\hline A drenaline & $<95$ & $\mathrm{pg} / \mathrm{mL}$ \\
\hline Total catecholamine & $242-1125$ & $\mathrm{pg} / \mathrm{mL}$ \\
\hline
\end{tabular}

Figures 


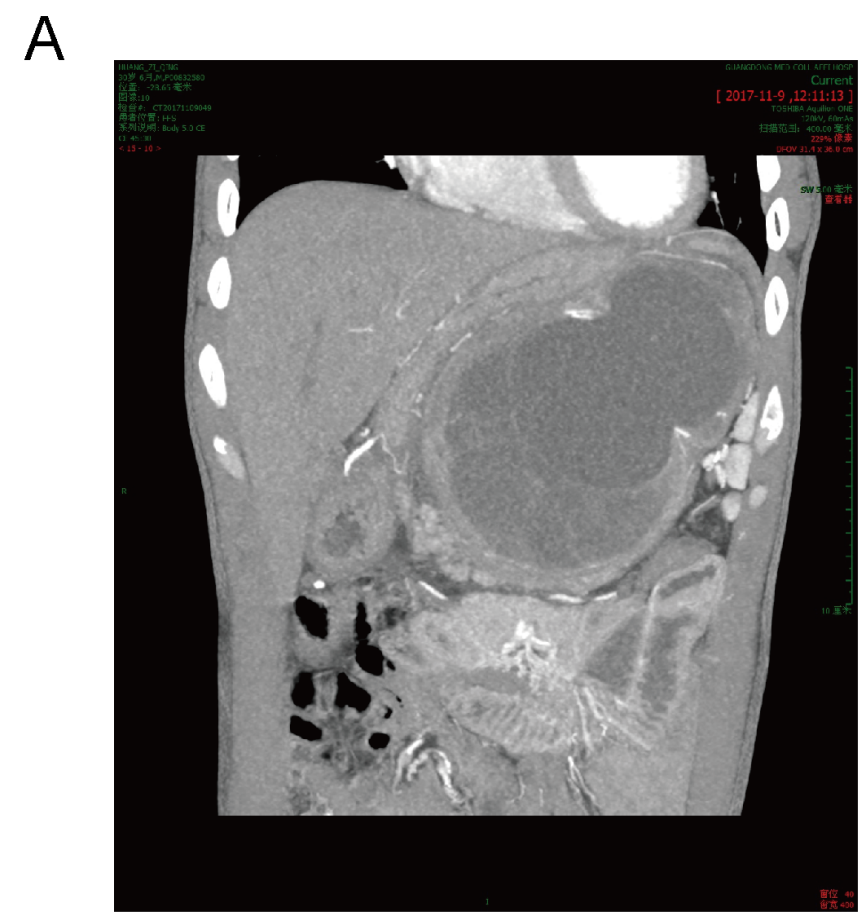

B

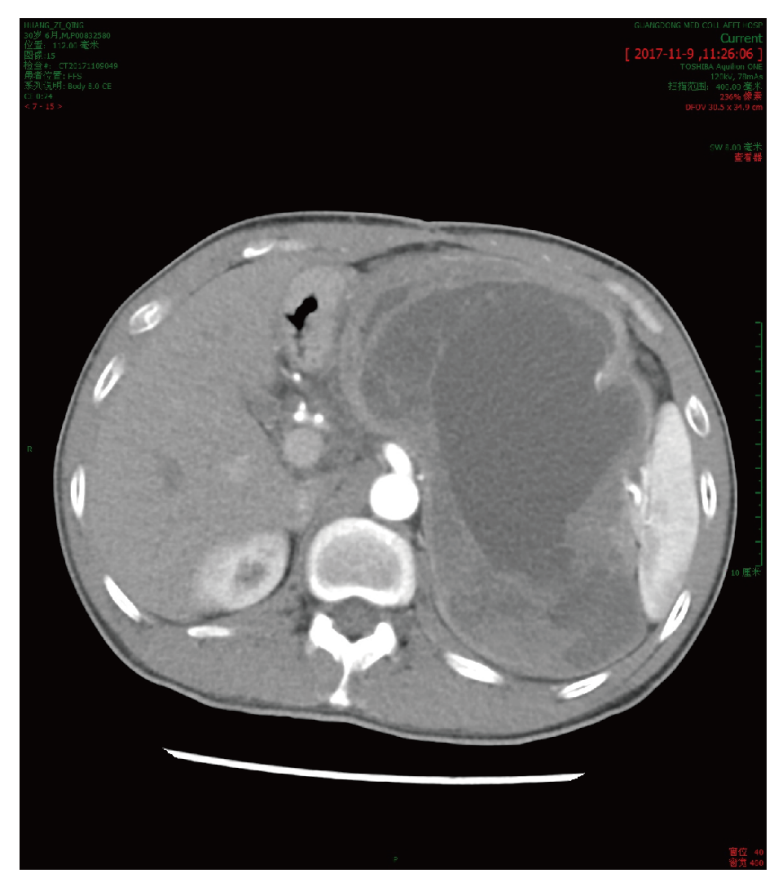

Dai et al. Figure 1

Figure 1

Abdomen CT images. (A) (B) 

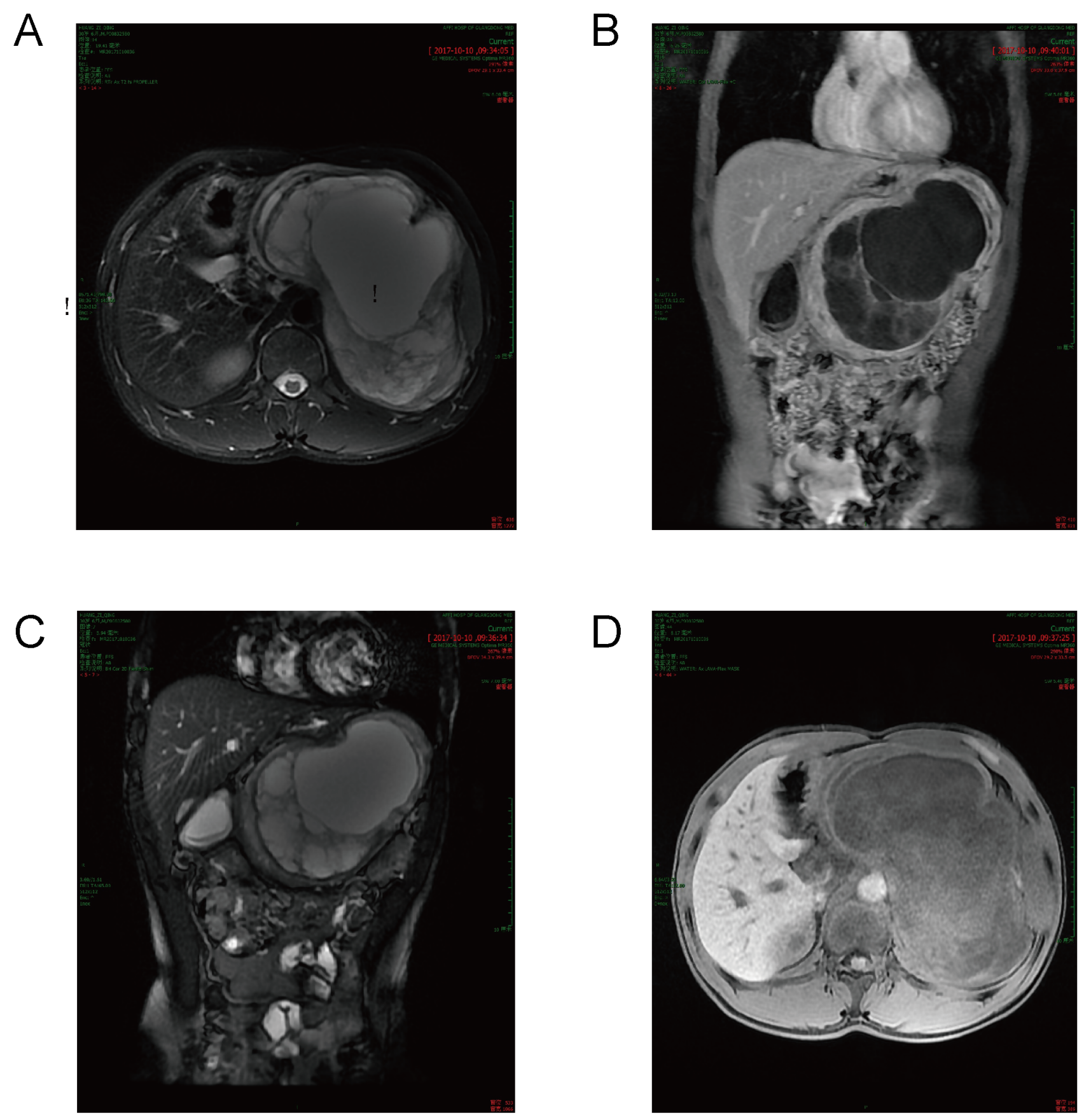

Dai et al. Figure 2

\section{Figure 2}

Abdomen MR images $₫ A$ and C:low signal in T1WI, B and D:high signal in T2WI区. (A) (B) (C) (D) 

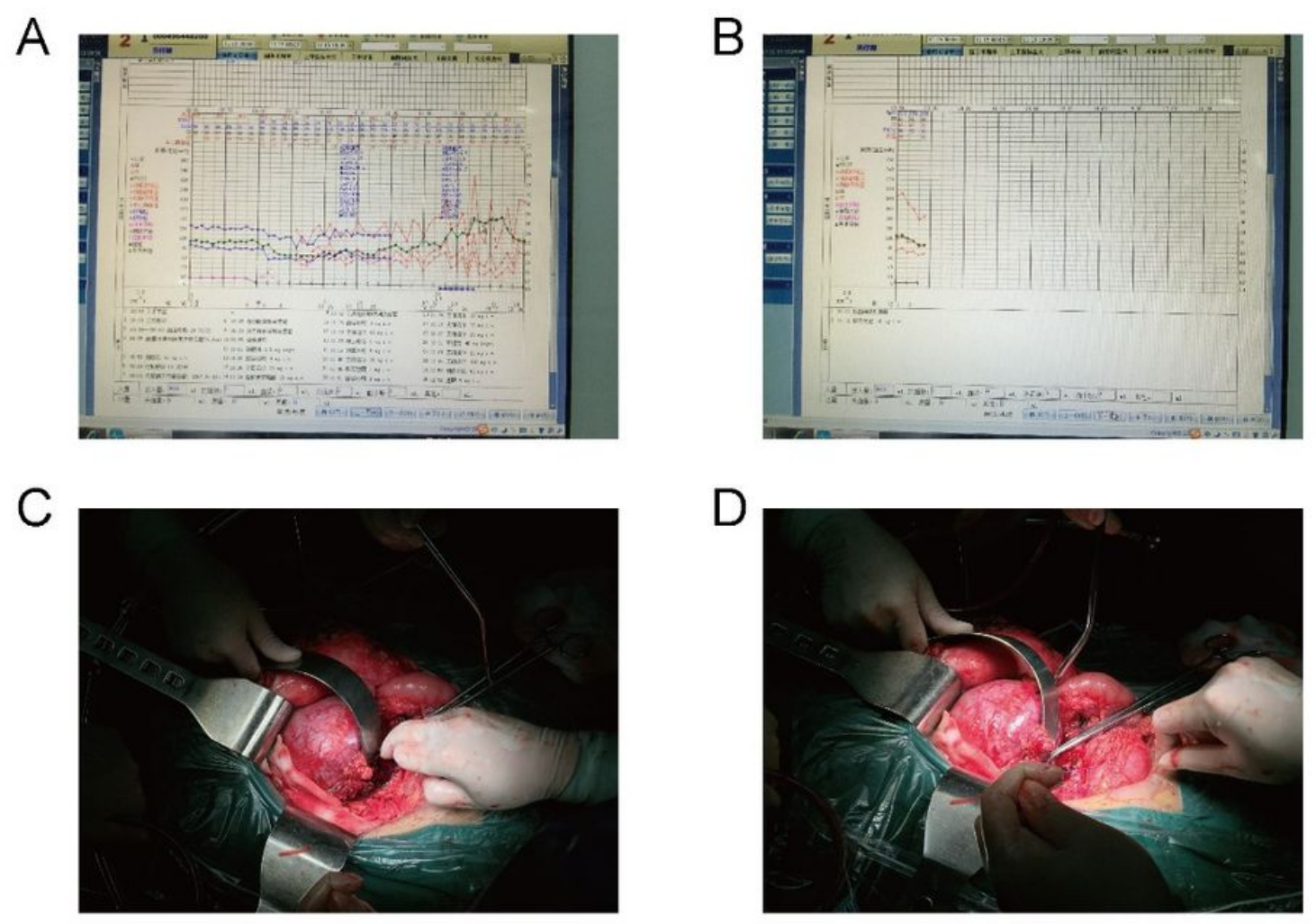

Dai et al. Figure 3

Figure 3

Real photos during the operation (A) (B) (C) (D) 

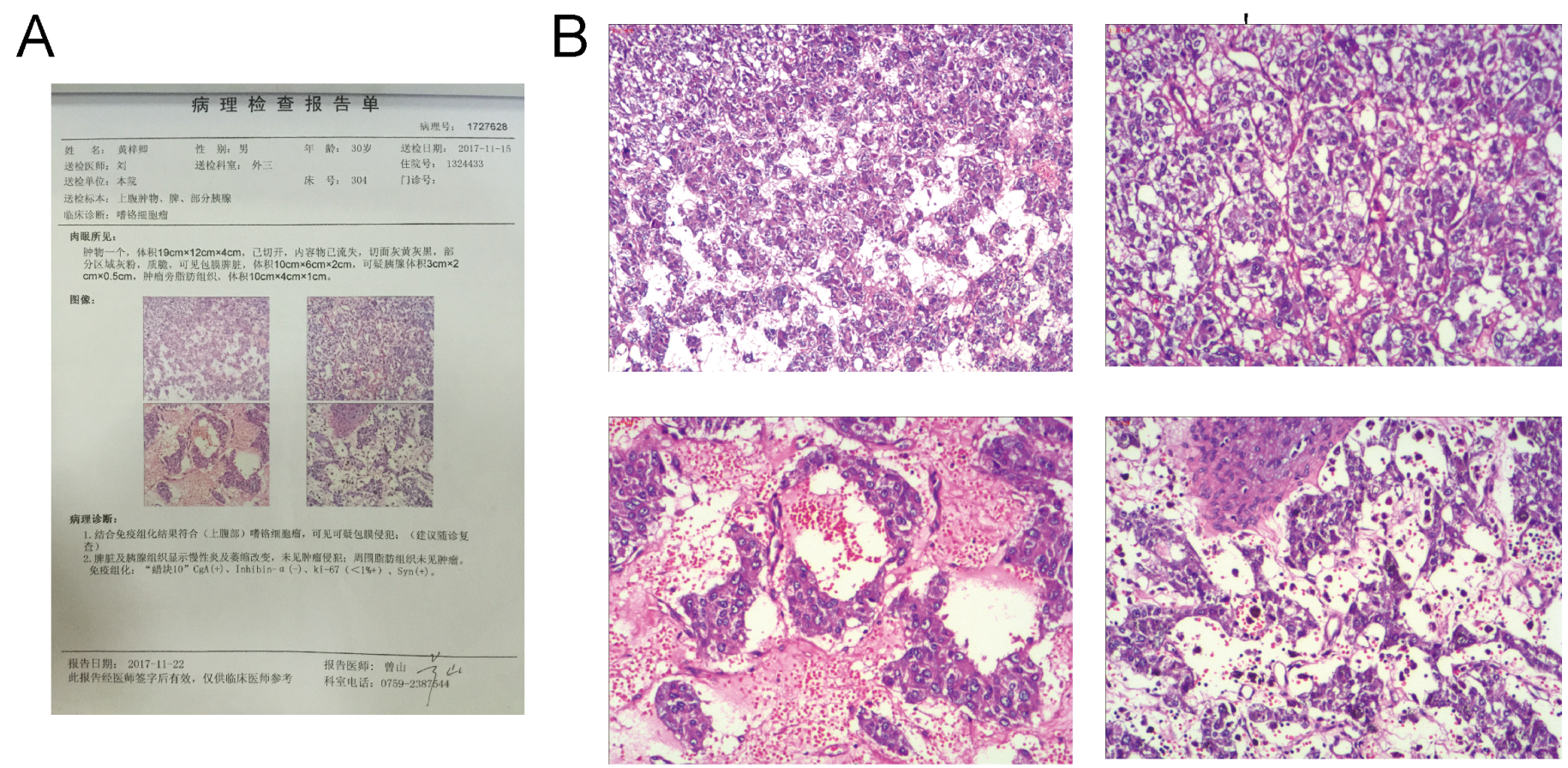

Dai et al. Figure 4

Figure 4

Pathology Report of the resected specimen (A) Pathology report (B) Immunohistochemistry:CgA(+), Inhibin-a(-), ki-67(<1\%+), Syn(+). Pheochromocytoma had the definite pathological diagnosis $₫ \times 200$ 